\title{
Evaluating Whole Blood Clotting in vitro on Biomaterial Surfaces
}

\author{
Roberta M Sabino ${ }^{1}$ and Ketul C Popat ${ }^{1,2,3, *}$
}

\author{
${ }^{1}$ School of Advanced Materials Discovery, Colorado State University, Fort Collins, USA; ${ }^{2}$ School of \\ Biomedical Engineering, Colorado State University, Fort Collins, USA; ${ }^{3}$ Department of Mechanical \\ Engineering, Colorado State University, Fort Collins, USA \\ *For correspondence: ketul.popat@colostate.edu
}

\begin{abstract}
[Abstract] Biomaterial-associated thrombosis is still a major concern for blood-contacting implants. After the medical device is implanted and comes in contact with blood, several complex reactions occur, which may lead to thrombus formation and failure of the device. Therefore, it is essential to evaluate the biomaterial interaction with the whole blood. Several studies have been reported in the literature that evaluate different steps in the coagulation cascade, such as protein adsorption, plasma activation, and platelet adhesion in vitro, however, evaluation of whole blood clotting on biomaterial surfaces is not widely reported. Here, a protocol to evaluate whole blood clotting in vitro on 2D biomaterials surfaces via a simple and fast hemolysis assay is presented. Whole human blood is placed onto the biomaterial surfaces and is allowed to clot for different time periods. After the specific time intervals, the surfaces are transferred into deionized (DI) water to release the free hemoglobin and the absorbance of this solution is measured. The absorbance value is proportional to the free hemoglobin concentration in the DI water due to lysis of red blood cells and gives an indirect correlation to the extent of blood clotting on the biomaterial surfaces. This protocol provides a fast, facile and effective method to measure the antithrombogenic properties of biomaterials.
\end{abstract}

Keywords: Blood clotting, Biomaterials, Blood-contacting implants, Thrombosis, Hemocompatibility test, Thrombogenicity

[Background] Investigating the blood clotting on medical devices is essential for successful development of biomaterials for implantable medical devices. Until now, no biomaterial surface has been truly able to prevent blood clotting (Sabino et al., 2019). After contact with blood, the implant surface gets an adsorbed layer of blood protein, which can further activate the coagulation cascade, leading to platelet adhesion and activation, and finally to the development of the fibrin mesh (Gorbet and Sefton, 2004). Many published methods to investigate the anti-thrombogenic properties of biomaterials focus on studying the early stages of blood clotting, such as protein adsorption, and platelet adhesion and activation. Although they are important to understand the interaction between blood and the implant surface, these studies do not provide significant information about the overall coagulation process (Damodaran et al., 2013; Simon-Walker et al., 2018; Obstals et al., 2018).

Preventing whole blood clotting on surfaces is crucial for long term success of blood-contacting implants. The formation of a fibrin clot is one of the latest stages of thrombosis, and this fibrin mesh traps the red blood cells (Leszczak et al., 2013). In this protocol, human blood is allowed to clot on Copyright $\odot 2020$ The Authors; exclusive licensee Bio-protocol LLC. 
biomaterial surface for up to $45 \mathrm{~min}$. When the surface is transferred to DI water, only the red blood cells that are not trapped in the fibrin mesh are dissolved in water and get lysed due to pressure change. Hemolysis is the rupture of the red blood cells, followed by the release of their components, such as hemoglobin. Thus, a higher amount of hemoglobin released indicates less blood clotting on the surface. Positive control with just blood in DI water is prepared and is considered the maximum hemoglobin release. The absorbance measured at a wavelength of $540 \mathrm{~nm}$ is directly proportional to the concentration of free hemoglobin in water (Sabino et al., 2019). Therefore, a higher absorbance value indicates higher hemoglobin concentration, which means less clotting on the biomaterial surface.

\section{Materials and Reagents}

1. $3.0 \mathrm{ml}$ vacuum blood tubes with no anticoagulant (BD Vacutainer, catalog number: 366703 )

2. 24-well plate (Greiner Bio-One CELLSTAR, catalog number: 662-160)

3. 96-well plate (Greiner Bio-One CELLSTAR, catalog number: 687-100)

4. Standard pipette tips with a volume capacity of $20 \mu$ land 1,000 $\mu$ l (Eppendorf, catalog numbers: 22491911 and 22491351)

5. Deionized (DI) water

6. Human blood

7. Biomaterial surface (average top area $\sim 25 \mathrm{~mm}^{2}$ )

8. Vacuum line

9. Marker (from any commercial source)

10. $70 \%$ ethanol

11. PBS (see Recipes)

\section{Equipment}

1. Microplate reader (BMG LABTECH, FLUOstar Omega)

2. Horizontal shaker (VWR, catalog number: 97109-890)

3. Measurement pipettes (Eppendorf, catalog numbers: ES-20F and ES-1000F)

4. Timer (from any commercial source)

5. Tweezers (from any commercial source)

6. Bench

\section{Software}

1. Software Omega and MARS Data Analysis (BMG LABTECH, https://www.bmglabtech.com/reader-control-software/)

2. Software Microsoft Excel or Origin (OriginLab, https://www.originlab.com/) 


\section{Procedure}

Note: All procedures are performed twice (using blood from two different donors) for three samples of each surface in the hood.

A. Biomaterial sterilization

1. Place biomaterial surfaces in each well of a 24-well plate. Prepare one plate for each time point $(15,30$ and $45 \mathrm{~min})$ and label them on the lid with the corresponding time point.

2. Add $500 \mu \mathrm{l}$ of ethanol for $5 \mathrm{~min}$ at room temperature.

3. Aspirate the ethanol and rinse the biomaterial surfaces three times with $500 \mu \mathrm{l}$ of PBS.

4. Aspirate the PBS and rinse the biomaterial surfaces once with $500 \mu \mathrm{l}$ of DI water.

5. Aspirate the DI water and let the biomaterial surfaces dry for at least $30 \mathrm{~min}$.

B. Adding blood to the biomaterial surface

1. Draw human whole blood in a vacuum tube with no anticoagulant.

2. Immediately after the blood draw, carefully open the vacuum tube and pipette $7 \mu \mathrm{l}$ of blood out of the tube and put onto each biomaterial surface (Figure 1).

3. Close the lid and start counting the timer for each plate (15, 30 and $45 \mathrm{~min})$.

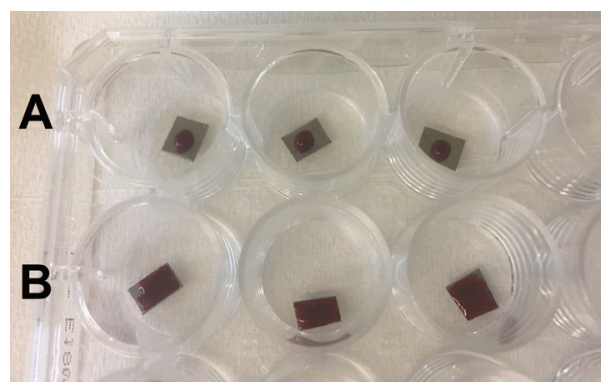

Figure 1. Whole blood on biomaterial surfaces. $7 \mu \mathrm{l}$ of blood are placed onto each surface and allowed to clot for 15,30 and $45 \mathrm{~min}$. Group A and B are different biomaterial surfaces being studied.

C. Preparing the positive control (maximum hemoglobin release)

1. Prepare a new 24-well plate with $500 \mu \mathrm{l}$ of DI water in 3 wells.

2. Add $7 \mu$ l of blood to each well with DI water (Figure $2 \mathrm{~A}$ ). 
A

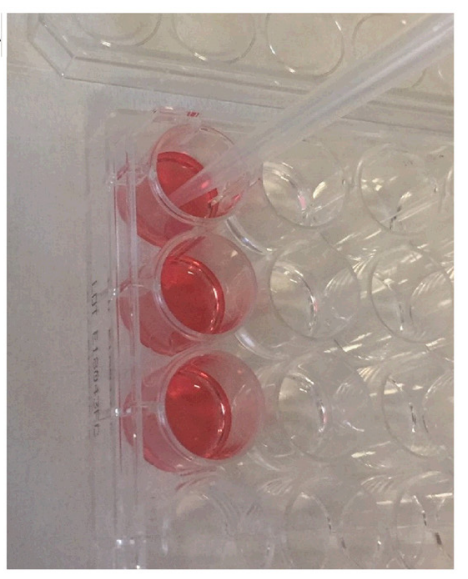

B

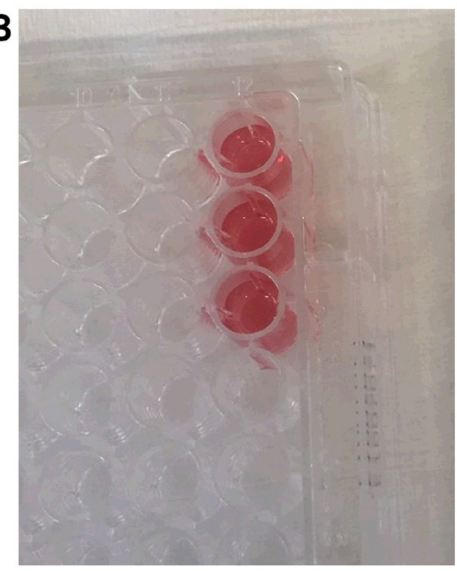

Figure 2. Positive Control. A. the maximum hemoglobin release is obtained adding $7 \mu \mathrm{l}$ of blood in $500 \mu \mathrm{l}$ of DI water. B. $200 \mu \mathrm{l}$ of each solution is transferred to a 96 -well plate to read the absorbance.

3. Place the plate on a horizontal shaker for $30 \mathrm{~s}$ (100 rpm).

4. Move the plate to a bench and wait for $5 \mathrm{~min}$ to release the free hemoglobin.

5. After $5 \mathrm{~min}$, pipette $200 \mu \mathrm{l}$ of the solution to a 96 -well plate (Figure 2B).

6. Read the absorbance using a microplate reader at a wavelength of $540 \mathrm{~nm}$.

D. Transferring samples to release hemoglobin

1. Prepare a new 24-well plate with $500 \mu \mathrm{l}$ of DI water in each well.

2. After 15 min, gently transfer the biomaterial surfaces from the first plate to a new well with DI water. Be careful while moving the biomaterial surfaces to not disturb the blood on the surface. The samples should under water after this step. If your samples are thicker, use more water in order to cover the whole with the blood.

3. Place the plate on a horizontal shaker for $30 \mathrm{~s}$ (100 rpm).

4. Move the plate to a bench and wait for $5 \mathrm{~min}$ to release the free hemoglobin (Figure 3).

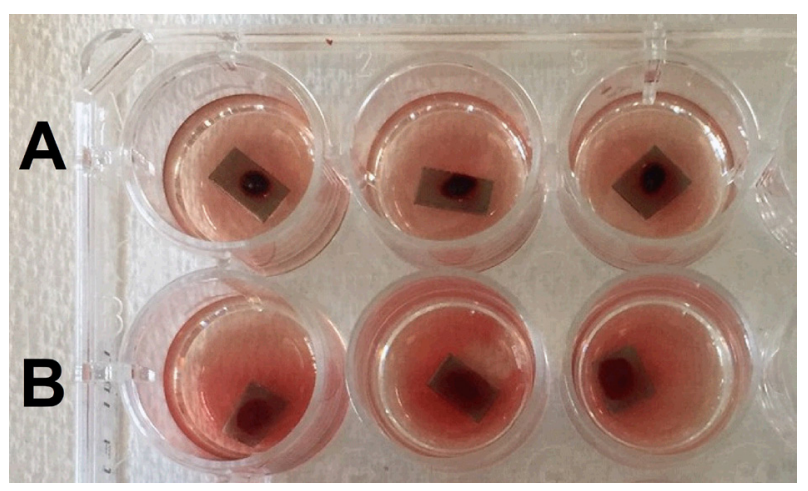

Figure 3. Different biomaterial surfaces, $A$ and $B$, in DI water to release the free hemoglobin. The lysis of red blood cells occurs due to pressure change and hemoglobin is released in DI water. 
E. Reading the absorbance

1. After $5 \mathrm{~min}$, pipette $200 \mu \mathrm{l}$ of the "water + hemoglobin released" solution to a 96-well plate. Change the pipette tip for each solution (Figure 4).

2. Read the absorbance using a microplate reader at a wavelength of $540 \mathrm{~nm}$.

3. Repeat Procedures $C$ and $D$ after 30 and $45 \mathrm{~min}$ for the second and third plates, respectively.

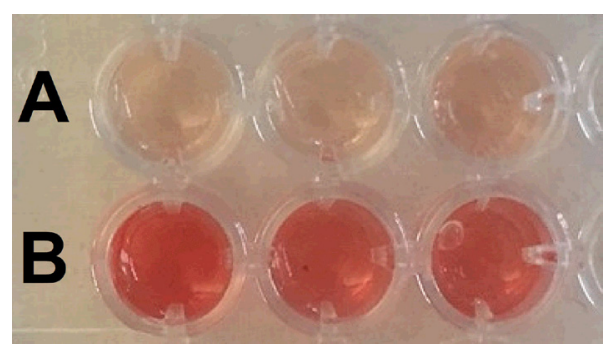

Figure 4. Solutions from different biomaterial surfaces, A and B (water + hemoglobin released). $200 \mu \mathrm{l}$ of each solution in Figure 3 is transferred to a 96-well plate to read the absorbance.

\section{Data analysis}

1. Absorbance values at $540 \mathrm{~nm}$ are read using a microplate reader. Export the data to an excel format file.

2. Any protocols involving blood are recommended to be performed at least twice using two different blood donors. However, in order to avoid donor-to-donor variability, only present the results obtained from one donor (Damodaran et al, 2013; Sabino et al., 2019).

3. Using Origin/Excel (or similar software), plot the mean/standard deviation of absorbance values from each group (Figure 5). Insert a line corresponding to the average absorbance measured for the positive control.

4. Conduct analysis of variance (ANOVA) for the experimental data using software Origin (or similar) at a $5 \%$ significance level $(P<0.05)$. 


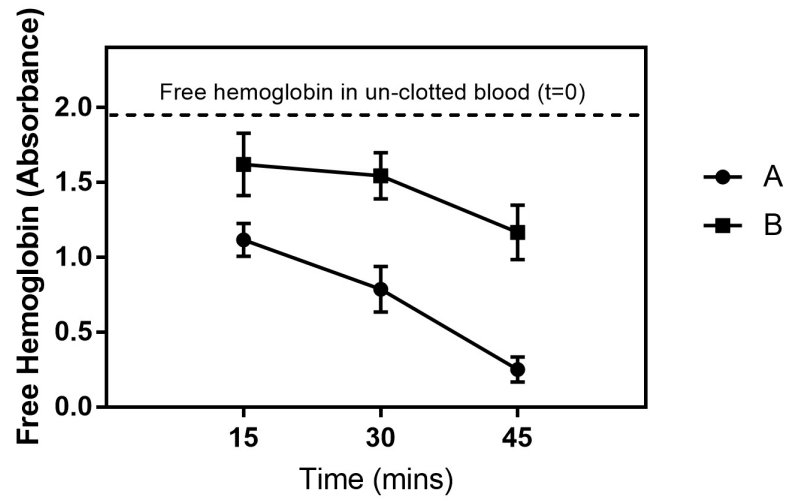

Figure 5. Free hemoglobin concentration values measured in terms of absorbance for different biomaterial surfaces after 15,30 and $45 \mathrm{~min}$. The results were significantly different for all time points. Group B shows significant higher hemoglobin concentration, which indicates that these surfaces significantly delay whole blood clotting in comparison with group A.

\section{Notes}

This protocol can be applied to evaluate the whole blood clotting in vitro of any biomaterial surface that is stable underwater. If the sample floats in water, use double-sided tape to attach the sample to the well bottom. Sterilization process may vary depending on the biomaterial surface.

\section{$\underline{\text { Recipes }}$}

1. $\operatorname{PBS}(10 \mathrm{x})$

Dissolve $100 \mathrm{ml}$ of 10x PBS in $900 \mathrm{ml}$ of DI water and autoclave or filter sterilize

\section{Acknowledgments}

This work was supported by National Heart, Lung and Blood Institute of the National Institutes of Health under award number R01HL135505 and R21HL139208. This protocol was adapted from previous publications from our group (Leszczak et al., 2013; Sabino et al., 2019).

\section{Competing interests}

The authors declare no conflict of interest.

\section{Ethics}

All experiments were conducted in agreement with the National Institutes of Health's "Guiding Principles for Ethical Research". Colorado State University Institutional Review Board approved the 
protocol $(17-7195 \mathrm{H}$, valid from $04 / 01 / 2017$ to $03 / 31 / 2020)$ for blood isolation from healthy participants. Whole human blood was acquired through venipuncture from healthy individuals, and formal consents were obtained from the donors.

\section{References}

1. Damodaran, V. B., Leszczak, V., Wold, K. A., Lantvit, S. M., Popat, K. C. and Reynolds, M. M. (2013). Anti-thrombogenic properties of a nitric oxide-releasing dextran derivative: evaluation of platelet activation and whole blood clotting kinetics. RSC Adv 3(46).

2. Gorbet, M. B. and Sefton, M. V. (2004). Biomaterial-associated thrombosis: roles of coagulation factors, complement, platelets and leukocytes. Biomaterials 25(26): 5681-5703.

3. Leszczak, V., Smith, B. S. and Popat, K. C. (2013). Hemocompatibility of polymeric nanostructured surfaces. J Biomater Sci Polym Ed 24(13): 1529-1548.

4. Obstals, F., Vorobii, M., Riedel, T., de Los Santos Pereira, A., Bruns, M., Singh, S. and Rodriguez-Emmenegger, C. (2018). Improving hemocompatibility of membranes for extracorporeal membrane oxygenators by grafting nonthrombogenic polymer brushes. Macromol Biosci 18(3).

5. Sabino, R. M., Kauk, K., Movafaghi, S., Kota, A. and Popat, K. C. (2019). Interaction of blood plasma proteins with superhemophobic titania nanotube surfaces. Nanomedicine 21: 102046.

6. Simon-Walker, R., Cavicchia, J., Prawel, D. A., Dasi, L. P., James, S. P. and Popat, K. C. (2018). Hemocompatibility of hyaluronan enhanced linear low density polyethylene for blood contacting applications. J Biomed Mater Res B Appl Biomater 106(5): 1964-1975. 